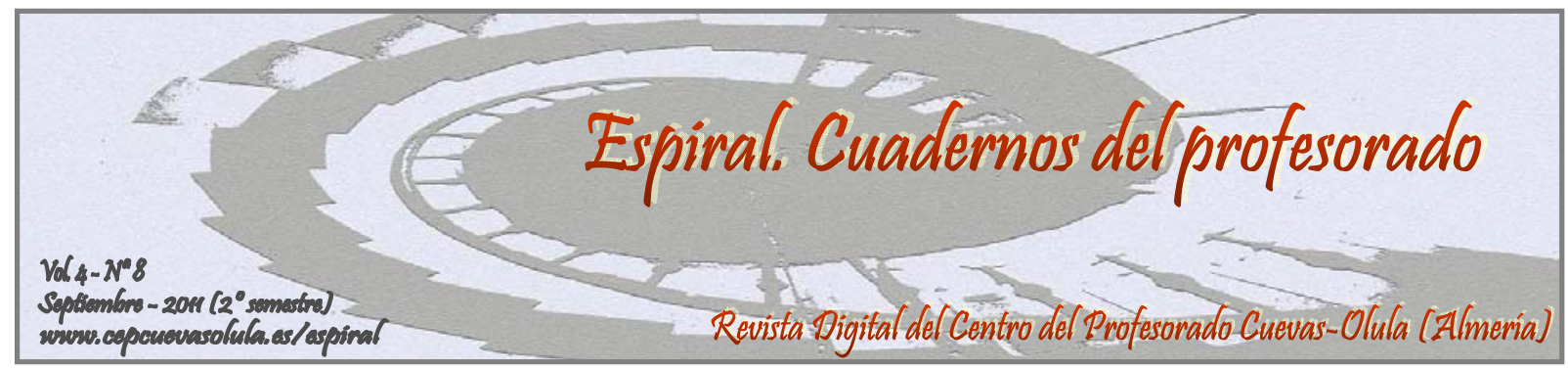

\title{
ORIENTACIONES DE META DE LOS JÓVENES ESCOLARES DEL COLEGIO EL BUEN PASTOR DE MURCIA
}

\section{GOAL ORIENTATION OF THE SCHOOLCHILDREN FROM THE BUEN PASTOR SCHOOL IN MURCIA}

\author{
Irene Castañón Rubio ${ }^{(1)}$, Nuria Rodríguez Suárez ${ }^{(1)}$ \\ y Antonio Granero-Gallegos ${ }^{(2)}$
}

\author{
${ }^{(1)}$ Facultad de Ciencias de la Salud, de la Actividad Física y del Deporte, Universidad Católica \\ San Antonio de Murcia, España Facultad de Humanidades, Universidad de Almería, España

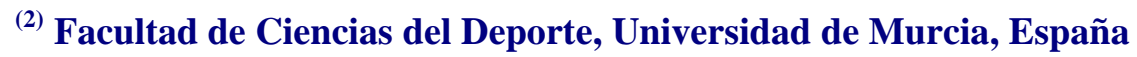

RESUMEN: El presente estudio analizó las Orientaciones de Meta de los jóvenes escolares de Educación Secundaria matriculados en $2^{\circ}, 3^{\circ}$ y $4^{\circ}$ curso, en sus clases de Educación Física. Para ello se han examinado las diferencias existentes en función de las variables sexo y edad; ya que dependiendo de las mismas, su motivación y orientación difiere, afectando en la clase de Educación Física. Se ha utilizado una muestra de 148 estudiantes que cursaban $2^{\circ}, 3^{\circ}$ ó $4^{\circ}$ de ESO en el Colegio El Buen Pastor de Murcia. El instrumento empleado fue el Cuestionario de Percepción de Éxito (POSQ). Para los resultados se ha realizado un cálculo de los principales estadísticos descriptivos, calculando la fiabilidad de las dos subescalas, a través del índice de consistencia interna de alfa de Cronbach, obteniendo como resultados mayores niveles en los escolares respecto a la orientación a la tarea y en cuanto a las diferencias por sexo, mayor porcentaje de disposición tanto al ego como a la tarea en la población masculina.

Palabras clave: Orientación de Meta, motivación, adolescente, Educación Física.

ABSTRACT: The next study analyzed the Goal Orientation of the schoolchildren of secondary school registered in $2^{\circ}, 3^{\circ}$ and $4^{\circ}$ course, in their Physical Education classes. For this, differences according to sex and age variables have been examined; as depending on them, their motivation and orientation differs, affecting in the Physical Education classes. A sample of 148 students from the high school has been used and they studied $2^{\circ}, 3^{\circ}$ and $4^{\circ}$ of secondary in the Buen Pastor School from Murcia. The instrument which was used was the Perception of Success Questionnaire: POSQ. For the results, a calculation of the main descriptive statistics have been made, calculating the two subscales reliability, through to index internal coefficient of Cronbach's alpha, getting as results higher levels in high schoolchildren on task orientation and as the differences for sex, higher disposition percentage as in the ego as in the task in male population

Key words: Goal Orientation, motivation, teenager, Physical Education. 
Castaño Rubio, I., Rodríguez Suárez, N., y Granero-Gallegos, A. (2011). Orientaciones de meta de los jóvenes escolares del colegio El Buen Pastor de Murcia. Espiral. Cuadernos del Profesorado, 4(8), 13-21. Disponible en: http://www.cepcuevasolula.es/espiral.

Fecha de recepción: 28/03/2011

Fecha de aceptación: 12/06/2011
Enviar correspondencia a: agranero@um.es

\section{1.- INTRODUCCIÓN}

Según Dosil (2004), la motivación es una variable psicológica que incita al sujeto hacia la práctica, orientación, mantenimiento y/o abandono de las actividades físicas/deportivas, y suele estar determinada por la asociación cognitiva que el individuo hace de las diferentes situaciones, en función de una serie de agentes (individuales, sociales, ambientales y culturales.

En la presente investigación estudiamos la Teoría Motivacional de las Metas de Logro en el ámbito docente en Educación Física; dicha teoría expone que el sujeto es guiado por unos objetivos hacia un fin (meta) que actúa de manera racional (Nicholls, 1984a). El individuo lucha por mostrar capacidad y destreza cuando se expone a contextos de logro (Dweck, 1986; Maehr y Braskamp, 1986; Nicholls, 1984) como pueden ser el educativo, deportivo y/o familiar, repercutiendo los mismos en su orientación, según Dweck (1999 en García, Cervelló, Jimenez, Iglesias y Santos-Rosa, 2005) "a esta tendencia a utilizar una u otra concepción de habilidad cuando se encuentra en dichos entornos se le conoce como Orientación Disposicional", existiendo a su vez dos tipos denominados Orientación a la Tarea y Orientación al Ego. El individuo también puede verse influido por el grado de madurez, nivel de maestría, personalidad, competitivo... afectando de nuevo en su Orientación Motivacional (Duda, 2005).

Varios trabajos recientes han demostrado que tanto en el contexto del deporte de competición como en el ámbito de la educación física, los "otros significativos", en este caso adultos significativos del entorno de los adolescentes, padres, entrenadores y profesores, afectan de modo directo e indirecto sobre las metas de logro que éstos adquieren (Cecchini, González, Carmona y Contreras, 2004; Duda, 2001; Ferrer-Caja y Weiss, 2000; Gutiérrez y García-Ferriol, 2001).

Así Nicholls (1989, en García, Cervelló, Jiménez, Iglesias y Santos-Rosa, 2005) decía que "la implicación al ego es una confección de habilidad fundamentada en los procesos de comparación social, donde el sujeto juzga su potencial con relación a otros, cuando el individuo emplea esta concepción de habilidad comparativa o diferenciada, la perspectiva de las metas de logro considera que el sujeto está implicado al ego. Por otro lado, cuando las acciones de los deportistas se dirigen hacia el aprendizaje y hacia una ejecución de maestría, las percepciones de habilidad son autoreferenciales y dependientes del progreso personal, dependiendo la percepción de éxito o el fracaso de la valoración subjetiva de si actuó con maestría, se aprendió, o si se mejora en una tarea, esta concepción de habilidad fue denominada por como implicación a la tarea". Se puede llegar a la conclusión de que cierto tipo de motivaciones, como la orientada al resultado, la de "ego" o la extrínseca pueden, en algún caso, resultar perjudiciales, afectando de forma negativa en la participación de la actividad, puesto que el deportista le otorga a elementos externos, que se escapan de su control, un valor determinante en el mantenimiento de dicha practica.

Los trabajos de Ntoumanis (2001) y Xiang y Lee (2002) son ejemplos de cómo la orientación hacia la tarea y los determinantes motivacionales intrínsecos determinan la continuidad en una actividad y la reducción de las tasas de abandono. En diferentes investigaciones se obtuvieron resultados que mostraban que los sujetos que perciben un clima motivacional en el que la demostración de la habilidad se basa en la mejora personal y en el esfuerzo, manifiestan un patrón significativamente más adaptativo que, aquellos que perciben un clima en el que la demostración de la habilidad se sustenta en la capacidad para resistir la comparación con los otros y en vencer a los demás. 
Dentro de este contexto deportivo encontramos que los chicos están más orientados al ego que las chicas y creen en mayor medida que ellas, que el éxito en el deporte depende de la capacidad y del uso de técnicas de engaño. Además, los chicos informan divertirse practicando deporte en mayor medida que las chicas, mientras que estas informan aburrirse con dichas prácticas en mayor medida que los chicos. (Castillo, Balaguer y Duda, 2002).

Se debe enfatizar los logros autoreferenciados que se relacionan con el dominio de la habilidad, la diversión, el esfuerzo y el interés por la actividad en sí misma, sin concederle ningún valor subordinado a los resultados objetivos de la competición en relación a los demás, ya que en la competición la orientación a la tarea resulta ser una experiencia más gratificante (Cecchini et al, 2004), algunas investigaciones han destacado que la orientación a la tarea se encuentra relacionada con un nivel de motivación intrínseca más elevado que la orientación al ego (Cervelló, Escartí y Balagué, 2000; Duda, Chi, Newton, Walling y Catley, 1995; Kim, Williams y Gill, 2003).

De esta orientación dependerá el motivo del abandono o la continuación de la práctica deportiva del adolescente, por lo que la orientación de meta de los jóvenes, es un estudio muy importante para conocer bien al alumno, su orientación de meta, y la influencia de los agentes psicosociales que van a influir principalmente en el joven, que serán: los padres y profesores, seguidamente serán los amigos y grupos de iguales (entornos de logro), repercutiendo en la toma de decisión y Orientaciones de Meta.

De manera que los alumnos orientados a la tarea presentarán mayor motivación intrínseca que los orientados al ego, ya que, el compromiso con una meta de tarea supone concebir la actividad a realizar como un fin en sí mismo. Con esta disposición, los alumnos se centrarán en el proceso de mejora de la tarea antes que en las consecuencias del resultado (aprobación social o recompensas), presentarán actitudes cooperativas, de esfuerzo y un mayor nivel de compromiso con la actividad. Por el contrario, el compromiso con una meta orientada al ego, disminuirá la motivación intrínseca porque la actividad se verá como un medio para conseguir otros fines, como contentar al profesor o a los padres, obtener prestigio social o cualquier otra motivación ajena a la propia tarea (Guitierrez y Escartí, 2006). Por ello, motivar adecuadamente o hacer que se motiven, es una preocupación constante de los entrenadores y profesores, con este planteamiento hemos realizado el presente estudio en el que investigamos las orientaciones de meta que tienen los escolares de ESO (hacia el ego o hacia la tarea), pues se consideran que son cuestiones de gran interés para el correcto planteamiento y funcionamiento de las clases de Educación Física.

En nuestro estudio hemos analizado la motivación en una muestra estudiantes de la Educación Secundaria Obligatoria (ESO) partiendo de la Teoría de las Metas de Logro. El objetivo de este trabajo es investigar las orientaciones de meta de los jóvenes escolares de la ESO del colegio El Buen Pastor de Murcia, analizando las diferencias existentes en función de las variables sexo y edad.

\section{2.- METODO}

\section{Muestra}

Se utilizó una población de 148 alumnos del Colegio El Buen Pastor (Murcia) pertenecientes a $2^{\circ}, 3^{\circ}$ y $4^{\circ}$ curso de la ESO. De los cuales obtuvimos una muestra de 59 alumnos de $2^{\circ}$ ESO $(39,9 \%), 46$ alumnos de $3^{\circ}$ ESO $(31,1 \%)$ y, finalmente 43 alumnos de $4^{\circ}$ ESO $(29,1 \%)$ quedando ésta compuesta por un total de 68 varones $(45,9 \%)$ y 80 mujeres $(54,1 \%)$, todos ellos de edades entre 13 y 17 años $(M=14,32 ; D T=1,50)$.

\section{Instrumentos}

El instrumento de la investigación ha sido el Cuestionario de Percepcion de Éxito (Perception of Success Questionarie: POSQ), utilizado para medir las Metas de Logro Disposicionales en las clases de Educación Física, diseñado por Roberts y Balagué $(1989,1991)$ y Roberts, Treasure y Balagué (1998). Se empleó para ello la versión validada al castellano (Cervelló, Escartí y Balagué, 1999). Este cuestionario consta de 12 ítems, de los cuales 6 miden la Orientación a la Tarea y 6 miden la Orientación al Ego. Las respuestas a los ítems están reflejadas en una escala tipo Likert con un rango 
de respuesta de 1 a 5 , en la que el 1 corresponde a totalmente en desacuerdo y el 5 a totalmente de acuerdo con el enunciado del ítem (En Educación Física siento que tengo éxito...). Estudios previos han mostrado la validez exploratoria y confirmatoria de la estructura factorial en dos subescalas en el ámbito escolar español (Cervelló y Santos-Rosa, 2000; Jiménez, 2001; Jiménez, Cervelló y Julián, 2001; Jiménez, Iglesias, Santos-Rosa y Cervelló, 2003), con $\alpha$ de .80 a .88 para la subescala Tarea y de .73 a .94 para la subescala Ego. En el presente trabajo, los resultados de fiabilidad del alfa de Cronbach demuestran una aceptable consistencia interna de las subescalas, tanto en la orientación disposicional al Ego $(\alpha>.85)$, como de la Tarea $(\alpha>.73)$.

\section{Procedimiento}

Para que alumnos y alumnas cumplimentasen este cuestionario se solicitó el permiso al centro escolar en el cuál se iba a realizar el estudio, así como a los diferentes tutores de los tres cursos mediante una reunión con los mismos, donde se les facilitó una versión del mismo. Además en esta junta se explicaron los objetivos de la investigación y cuál era el procedimiento a seguir. El cuestionario fue administrado por los investigadores en el centro, en las horas de las tutorías. Todos los participantes fueron informados del objetivo de estudio, voluntariedad y confidencialidad de las respuestas y manejo de datos, que no había respuestas correctas o incorrectas, solicitándoles al inicio de este que contestara con la máxima sinceridad y honestidad.

\section{Análisis estadístico}

Se ha realizado un cálculo de los principales estadísticos descriptivos, media $(M)$ y desviación típica (DT), calculando la fiabilidad de las dos subescalas, a través del índice de consistencia interna de alfa de Cronbach. Se realizaron las pertinentes pruebas de normalidad y homocedasticidad para asegurar la homogeneidad de la varianza. Se han calculado los índices de asimetría y curtosis siendo, en general, próximos a cero y $<2$, tal y como recomiendan Bollen y Long (1994), lo que indica semejanza con la curva normal de forma univariada y permite utilizar técnicas factoriales. En el análisis de las diferencias por género y edad se ha utilizado la prueba de la $T$ de Student para muestras independientes. Para todo ello se empleó el paquete estadístico SPSS en su versión 17.0 para Windows.

\section{3.- RESULTADOS Y DISCUSIÓN}

\section{Análisis descriptivo}

En la tabla 1 se exponen los valores descriptivos de cada una de las variables de la investigación. Respecto a las medias, en la Percepción del Éxito se observa un mayor valor en el factor tarea $(M=$ $3.80 ; D T=.76)$ que en el ego $(M=3.30 ; D T=1.00)$.

Tabla 1. Media (M), desviación típica (DT), asimetría y curtosis de las subescalas del Cuestionario de Percepción de Éxito (POSQ).

\begin{tabular}{|c|c|c|c|c|}
\hline Dimensiones & $M$ & $D T$ & Asimetría & Curtosis \\
\hline \multicolumn{5}{|c|}{ Percepción del éxito (POSQ) } \\
\hline I. Ego & 3.30 & 1.00 & -.054 & -.746 \\
\hline II Tarea & 3.80 & .76 & -.609 & .544 \\
\hline
\end{tabular}

\section{Análisis inferencial}

\section{Diferencias según género y edad}

Para hallar las diferencias significativas según el género y la edad del alumnado se llevó a cabo la Prueba $T$ de Student para muestras independientes, teniendo en cuenta el valor de la Prueba de Levene, de homogeneidad de las varianzas, dado que son muestras independientes. Los datos más relevantes y que hacen referencia a la media $(M)$ y desviación típica $(D T)$ se recogen en la tabla 2 , mientras que en la tabla 3 se reflejan los datos de la Prueba $T$ de igualdad de medias. En esta tabla 3 se observa que no se hallaron diferencias significativas en función de la edad, pero sí según el género. 
Tabla 2. Prueba $T$ de Student para muestras independientes. Estadísticos según género y edad.

\begin{tabular}{lccccccccc}
\hline & \multicolumn{4}{c}{ Sexo } & \multicolumn{5}{c}{ Edad } \\
\cline { 2 - 10 } & \multicolumn{2}{c}{ Chicos } & \multicolumn{2}{c}{ Chicas } & \multicolumn{2}{c}{ De 13 a 14 años } & \multicolumn{1}{c}{ 15 y 16 años } \\
\hline \multicolumn{1}{c}{ Dimensiones } & $M$ & $D T$ & $M$ & $D T$ & $M$ & $D T$ & $M$ & $D T$ \\
\hline Percepción del éxito (POSQ) & & & & & & & & & \\
I. Ego & 3.71 & .94 & 2.95 & .93 & 3.26 & 1.04 & 3.35 & .96 \\
II. Tarea & 4,10 & .63 & 3.54 & .76 & 3.83 & .76 & 3.77 & .77 \\
\hline
\end{tabular}

Respecto al POSQ, en el primer factor (Ego), indicar que $F=.092 ; \mathrm{p} \leq .76$, por lo que se asume que las varianzas son iguales: $t(146)=-4.91 ; p \leq .000$; por consiguiente, existe diferencia de medias entre los chicos y las chicas, respecto a la orientación disposicional al ego, con datos muy por encima de la media entre los varones. En el factor Tarea, $F=2.46 ; p \leq .12$, por lo que se asume que las varianzas son iguales: $t(144)=-4.77 ; p \leq .000$; por tanto, también existen diferencias significativas de medias entre los chicos y las chicas, que son las que presentan datos por encima de la media.

Tabla 3. Prueba $T$ de Student para muestras independientes, según género y edad.

\begin{tabular}{lccccccc}
\hline & \multicolumn{3}{c}{$\begin{array}{c}\text { Prueba } \boldsymbol{T} \text { para la igualdad de } \\
\text { medias según sexo }\end{array}$} & \multicolumn{2}{c}{$\begin{array}{c}\text { Prueba } \boldsymbol{T} \text { para la igualdad de } \\
\text { medias según edad }\end{array}$} \\
\cline { 2 - 8 } Dimensiones & $t$ & $p$ & $\begin{array}{c}\text { Diferencia } \\
\text { medias }\end{array}$ & $t$ & $p$ & $\begin{array}{c}\text { Diferencia } \\
\text { medias }\end{array}$ \\
\hline $\begin{array}{l}\text { Percepción del éxito (POSQ) } \\
\text { I. Ego }\end{array}$ & -4.91 & .000 & -.75 & -.531 & .596 & -.90 \\
$\quad$ II. Tarea & -4.77 & .000 & -.56 & -.402 & .688 & .05 \\
\hline
\end{tabular}

\section{Análisis de regresión}

Se ha realizado un análisis de regresión múltiple para comprobar el valor predictivo de las variables género y edad sobre la percepción del éxito (POSQ) en el alumnado investigado (tabla 4). Los resultados pusieron de manifiesto que el género no fue predictor de la orientación de percepción del éxito en la población estudiada. En cambio, el género se ha mostrado como predictor significativo e importante, tanto en el ego $(\beta=.75 ; p \leq .000)$, con un $14.5 \%$ de la varianza explicada, como en la tarea $(\beta=.56 ; p \leq .000)$, con un $13.6 \%$ de la varianza explicada.

Tabla 4. Correlaciones, pesos beta estandarizados $(ß)$ y varianza total explicada $\left(R^{2)}\right.$ para cada factor, para el género y la edad como predictores de la percepción del éxito.

\begin{tabular}{lcccccccc}
\hline \multicolumn{1}{c}{ Variables } & \multicolumn{3}{c}{ Género } & \multicolumn{4}{c}{ Edad } \\
\hline \multicolumn{1}{c}{ Percepción del éxito (POSQ) } & $\boldsymbol{F}$ & $\boldsymbol{B}$ & $\boldsymbol{R}^{2}$ & $\boldsymbol{p}$ & $\boldsymbol{F}$ & $\boldsymbol{B}$ & $\boldsymbol{R}^{2}$ & $\boldsymbol{p}$ \\
$\quad$ I. Ego & & & & & & & & \\
II. Tarea & 24.12 & .75 & .142 & .000 & .28 & .09 & .007 & .596 \\
\hline
\end{tabular}

Nota.- Se realizaron análisis separados para cada factor.

\section{4.- DISCUSIÓN Y CONCLUSIONES}

El primer objetivo planteado en esta investigación ha sido analizar las Metas de Logro de los escolares del Colegio El Buen Pastor de Murcia. Nuestros resultados exponen valores más elevados en el factor tarea, en contraposición de los valores más bajos que corresponden al factor ego.

Todo lo reflejado anteriormente, avala la afirmación de que ambas orientaciones motivacionales son ortogonales entre sí (Cervelló, Escartí y Balagué, 1999), siendo esperanzadores estos resultados afectando de forma positiva al fomento de la adherencia deportiva, debido a que un individuo que presente tendencia positiva hacia la disposición a la tarea o la maestría, juzga su potencial mediante un proceso de comparación con él mismo, siendo su percepción de habilidad autorreferencial. Aquellos individuos que presentan una disposición motivacional orientada a la tarea muestran mayor nivel motivación de carácter intrínseco, favoreciendo de esta forma que el sujeto 
sienta mayor diversión y manifiesten mayor interés por la práctica deportiva (Cecchini, Méndez y Muñiz, 2002; Walling, Duda y Crawford, 2002) ya que son las principales características de la motivación intrínseca, aumentando la conducta positiva de conservación y unos patrones motivacionales afectivos y adaptativos (Cecchini, González, Carmona y Contreras, 2004).

Por el contrario, aquellos individuos que presentan una orientación disposicional hacia el ego, persiguiendo alcanzar estatus social y popularidad (Holgado, Navas y López-Núñez, 2010) empleando como fuente de información acerca de su capacidad la comparación externa (Cervelló, Escartí y Balagué, 1999; Treasure y Roberts, 1995), manifestarán mayor probabilidad de abandono prematuro (Vallerand y Losier, 1999) debido a que pueden obtener una mala autopercepción de su competencia y encortar pequeñas dificultades en la actividad (Castillo, Balaguer y Duda, 2002; Cervelló y SantosRosa, 2001) por lo que resultados deportivos no se corresponden con las expectativas y fracasan (Lochbaum y Roberts, 1993).

A tenor de todo lo expuesto, mencionar que la orientación al ego no siempre es perjudicial, ya que este tipo de motivación combinado con un nivel alto de orientación a la tarea se asocia con altos niveles de motivación (Wang y Biddle, 2001).

Debido a todo lo presentado anteriormente y considerando la Educación Física como un contexto importante para los niños, donde realizan procesos de comparación social con sus iguales, debemos tratar de crear climas motivacionales orientados hacia la tarea desde edades tempranas, ya que en la etapa de la adolescencia queda asentada la personalidad del niño, siendo mucho mas difícil modificar dichos patrones, que ya fueron consolidados. Esto contribuirá a que la práctica en las clases sea una experiencia positiva, evitando el fracaso, estrés y desmotivación, prolongando de esta manera la implicación de los escolares en las actividades deportivas y en la clase de Educación Física.

El segundo objetivo que fijamos fue analizar las diferencias motivacionales existentes en función de las variables sociodemográficas, sexo y edad. En cuanto a la primera de las variables, los resultados exponen diferencias significativas entre los hombres y las mujeres en las distintas orientaciones disposicionales (al ego y a la tarea). Los varones alcanzan niveles más elevados en la orientación disposicional al ego, resultados que coinciden con los hallados en otros estudios donde se ha utilizado la misma escala (Gano-Overway y Duda, 2001; González, Cecchini, Llavona y Vázquez, 2010; Hanrahan y Biddle, 2002; Kavussanu y Roberts, 2001) y en otros donde la escala utilizada ha sido la Task and Ego Orientation in Sport Questionnaire (TEOSQ, Castillo, Balaguer y Duda, 2000, 2002; Hanrahan y Cerin, 2009; Moreno, Cervelló y González-Cutre, 2008). De esta misma forma vuelven a ser los hombres los que manifiestan mayor porcentaje en la orientación a la tarea. Hanrahan y Cerin (2009) defienden que el sexo es un predictor de las orientaciones de metas disposicionales y regulador del nexo existente entre el nivel de participación y el estilo atribucional.

Con respecto a la edad, no encontramos resultados estadísticamente significativos en ninguna de las orientaciones disposicionales, no obstante, aunque en nuestro estudio no hemos hallado diferencias significativas en relación a la edad, otros estudios avalan que son los sujetos en edad mas temprana, los que presentan mayores niveles de orientación hacia el ego, ya que absorben mayor influencia del entorno (padres, entrenador, compañeros), por lo que podría estar influida por la percepción de la orientación motivacional percibida de los otros significativos, en un intento de responder a las expectativas que él percibe. Por todo ello entendemos que la relación que ejerce el ambiente con la orientación motivacional del deportista influye, ya que tiende a actuar conforme a lo que percibe (Ames y Archer, 1988; Nicholls, 1989; Escartí y García-Ferriol, 1993; Cervelló, Escartí y Balagué, 1999; Cervelló y Santos-Rosa, 2000).

\section{LIMITACIONES}

Finalmente, hay que resaltar que la investigación presenta ciertas limitaciones, en cuanto al tamaño de la muestra, lo que condiciona la generalización de los resultados. Por ello, los resultados de esta investigación deben ser considerados como preliminares y necesitarían ser replicados en un futuro. 


\section{AGRADECIMIENTOS}

Agradecemos a los alumnos del Colegio Buen Pastor de Murcia, así como todo el claustro de profesores y padres de alumnos toda la ayuda mostrada en la realización de nuestra investigación.

\section{5.- REFERENCIAS BIBLIOGRÁFICAS}

Ames, C., y Archer, J. (1988). Achievement goals in the classroom: Students' learning strategies and motivation processes. Journal of Educational Psychology, 80, 260- 267.

Bollen, K., y Long, J.S. (1994). Testing structural equation models. Newbury Park, CA: Sage.

Castillo, I., Balaguer, I., y Duda, J.L. (2000). Las orientaciones de meta y los motivos de práctica deportiva en los jóvenes deportistas valencianos escolarizados. Revista de Psicología del Deporte, 9(1-2), 37-50.

Castillo, I., Balaguer, I. y Duda, J.L. (2002). Las perspectivas de meta de los adolescentes en el contexto deportivo. Psicothema, 14 (2), 280- 287.

Cecchini, J.A., González, C., Carmona, A.M., y Contreras, O. (2004). Relaciones entre clima motivacional, la orientación de meta, la motivación intrínseca, la auto-confianza, la ansiedad y el estado de ánimo en jóvenes deportistas. Psicothema, 16(1), 104-109.

Cecchini, J.A., Méndez, A., y Muñiz, J. (2002). Motives for practicing sport in spanish schoolchildren. Psicothema, 14(3), 523-531.

Cervelló, E., y Santos-Rosa, F.J. (2001). Motivation in sport: and achievement goal perspective in young Spanish recreational athletes. Perceptual and Motor Skills, 92, 527-534.

Cervelló, E.M., y Santos-Rosa, F.J. (2000). Motivación en las clases de educación

Cervelló, E., Escartí, A., y Balagué, G. (1999). Relaciones entre la orientación de meta disposicional y la satisfacción con los resultados deportivos, las creencias sobre las causas de éxito en deporte y la diversión con la práctica deportiva. Revista de Psicología del Deporte, 8(1), 7-19.

Cervelló, E., Escartí, A., y Balagué, G. (2000). Relaciones entre la orientación de meta disposicional y la satisfacción con los resultados deportivos, las creencias sobre las causas de éxito en deporte y la diversión con la práctica deportiva. Revista de Psicología del Deporte, 10, 2-16.

Cervelló, E.M., y Santos-Rosa, F.J. (2000). Motivación en las clases de Educación Física: un estudio de la perspectiva de las metas de logro en el contexto educativo. Revista de Psicología del Deporte, 9, 51-70.

Dosil, J. (2004). Motivación: "motor" del deporte. Psicología de la actividad física y el deporte (pp. 127-153). Madrid: Mc Graw Hill.

Duda, J.L. (2001). Achievement goal reserach in sport: Pushing the boundaries and clarifying some misunderstandings. En G. C. Roberts (Ed.), Advances in motivation in sport and exercise (pp.129-182). Champaign, IL: Human Kinetics.

Duda, J.L. (2005). A self determination approach to predicting exercise engagement and associated well-being. Ponencia del $9^{\circ}$ Congreso Europeo de Psicología. Granada (3-8 de Julio): Consejo General del Colegio Oficial de Psicólogos- E.F.P.A.

Duda, J.L., Chi, L., Newton, M.L., Walling, M.D., y Catley, D. (1995). Task and ego orientation and intrinsic motivation in sport. International Journal Sport Psychology, 26, 40-63.

Dweck, C. S. (1986). Motivational processes affecting learning. American Psychologist, 41, 1040-1048. educativo. Revista de Psicología del Deporte, 9, 51-70.

Escartí, A., y García- Ferriol, A. (1993). El grupo de iguales y la motivación deportiva. En S. Barriga; J.L. Rubio (Eds.). Aspectos psicosociales del ambiente, la conducta deportiva y el fenómeno turístico, (pp. 171178). Sevilla: Eudema.

Ferrer-Caja, E., y Weiss, M. R. (2000). Predictors of intrinsic motivation among adolescent students in physical education. Research Quarterly for Exercise and Sport, 71, 267-279.

física: un estudio de la perspectiva de las metas de logro en el contexto

Gano-Overway, L.A., y Duda, J.L. (2001). Personal theories of achievement motivation among African and White Mainstream American athletes. International Journal of Sport Psychology, 32, 335-354.

García, T., Cervelló, E., Jiménez, R., Iglesias, D., y Santos-Rosa, F.J. (2005). La implicación motivacional de jugadores jóvenes de fútbol y su relación con el estado de flow y la satisfacción en competición. Revista de Psicología del Deporte, 14(1), 21-42. 
González, C., Cecchini, J.A., Llavona, A., y Vázquez, A. (2010). Influencia del entorno social y el clima motivacional en el autoconcepto de las futbolistas asturianas. Aula Abierta, 38(1), 25-36.

Gutierrez, M., y Escartí, A. (2006). Influencia de padres y profesores sobre las orientaciones de meta de los adolescentes y su motivación intrínseca en educación física. Revista de Psicología del Deporte, 15(1), 23-35.

Gutiérrez, M., y García-Ferriol, A. (2001). El entorno escolar-familiar y la práctica deportiva en la adolescencia: una aproximación empírica. En J. Dosil (Ed.), Psicología y deporte de iniciación (pp. 35-55). Ourense: Gersam.

Hanrahan, S.J., y Biddle, S.J.H. (2002). Measurement of achievement orientations: Psychometric measures, gender, and sport differences. European Journal of Sport Science, 2(5), 1-12.

Hanrahan, S.J., y Cerin, E. (2009). Gender, level of participation, and type of sport: differences in achievement goal orientation and attributional style. Journal of Science and Medicine in Sport, 12, 508-512.

Holgado, F., Navas, L., y López-Núñez, M. (2010). Goal orientations in sport: a causal model. European Journal of Education and Psychology, 3(1), 19-32.

Jiménez, R. (2001). Un estudio de la coeducación y los comportamientos de disciplina en las clases de Educación Física desde la perspectiva de las metas de logro: Análisis de las diferencias en función del género y la edad. Memoria de Docencia e Investigación. Cáceres: Universidad de Extremadura.

Jiménez, R., Cervelló, E.M., y Julián, J. (2001). Un estudio de las diferencias en la coeducación y los comportamientos de disciplina en función del género y las etapas educativas de los alumnos/as en las clases de Educación Física. Comunicación presentada en el IV Congreso Internacional sobre la enseñanza de la Educación Física y el Deporte Escolar, Santander.

Jiménez, R., Iglesias, D., Santos-Rosa, F.J., y Cervelló, E.M. (2003). Análisis de la relación entre el clima motivacional, las orientaciones de meta y la igualdad de trato en función del género en las clases de Educación Física. Comunicación presentada en el IX Congreso Nacional de Psicología de la Actividad Física y el Deporte "Perspectiva Latina”, León.

Kavussanu, M., y Roberts, G.C. (2001). Motivation in physical activity contexts: The relationship of perceived motivational climate to intrinsic motivation and self-efficacy. Journal of Sport and Exercise Psychology, 18, 264-280.

Kim, B.J., Williams, L., y Gill, D.L. (2003). A cross-cultural study of achievement orientation and intrinsic motivation in young USA and Korean athletes. International Journal of Sport Psychology, 34, 168-184.

Lochbaum, M., y Roberts, G.C. (1993). Goal orientations and perceptions of the sport experience. Journal of Sport and Exercise Psychology, 15(2), 160-171.

Maehr, M.L., y Braskamp, L.A. (1986). The motivation factor. A theory of personal investment. Lexington, M A: Lexington Books.

Moreno, J.A., Cervelló, E., y González-Cutre, D. (2008). Relationships among goal orientations, motivational climate and flow in adolescent athletes: differences by gender. The Spanish Journal of Psychology, 11(1), 181-191.

Nicholls, J.G. (1984a). Achievement motivation: Conceptions of ability, subjective experience, task choice, and performance. Psychological Review, 91, 328-346.

Nicholls, J. G. (1984b). Conceptions of ability and achievement motivation. In R. Ames y C. Ames (Eds.), Research on motivation in education: student motivation (Vol. I, pp. 39- 73). New York: Academic Press.

Nicholls, J.G. (1989). The competitive ethos and democratic education. Cambridge, MASS: Harvard University Press.

Ntoumanis, N. (2001). A self-determination approach to the understanding of motivation in physical education. British Journal of Educational Psychology, 71, 225-242.

Roberts, G.C., y Balagué, G. (1989). The development of a social-cognitive scale in motivation. Seventh World Congress of Sport Psychology. Singapore, Republic of Singapore.

Roberts, G.C., y Balagué, G. (1991). The development and validation of the Perception of Success Questionnaire. FEPSAC Congress. Cologne, Germany.

Roberts, G.C., Treasure, D.C., y Balagué, G. (1998). Achievement goals in sport: the development and validation of the Perception of Success Questionnaire. Journal of Sport Sciences, 16, 337-347. 
Treasure, D., y Roberts, G. (1995). Applications of achievement goal theory to physical education: implications for enhancing motivation. Quest, 47, 475-489.

Vallerand, R.J., y Losier, G.F. (1999). Self-determined motivation and sportsmanship orientations: An assessment of their temporal relationship. Journal of Sport and Exercise Psychology, 16, 229-245.

Walling, M.D., Duda, J.L., y Crawford, T. (2002). Goal orientations, outcome, and responses to youth competition among high/low perceived ability athletes. International Journal of Sport Psychology, 37(2), 115-122.

Wang, C.K.J., y Biddle, S.J.H. (2001). Young people's motivational profiles in physical activity: A cluster analysis. Journal of Sport and Exercise Psychology, 23, 1-22.

Xiang, P., y Lee, A. (2002). Achievement goals, perceived motivational climate, and students' self-reported mastery behaviours. Research Quarterly for Exercise y Sport, 73 (1), 58-65.

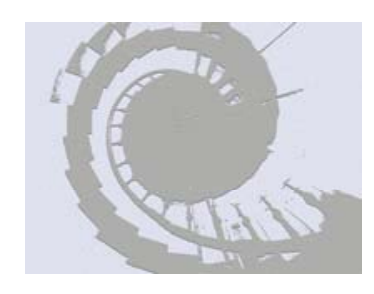

